\title{
ECHO's ECHO: Overcoming modern healthcare operational challenges with provider-to-provider video communication
}

\author{
Amber B. Moore*1,2, Lewis A. Lipsitz ${ }^{2,3}$ \\ ${ }^{1}$ Massachusetts General Hospital, Boston, United States \\ ${ }^{2}$ Harvard Medical School, Boston, Mass, United States \\ ${ }^{3}$ Beth Israel Deaconess Medical Center, Boston, Mass, United States
}

Received: March 1, 2020

DOI: $10.5430 /$ jha.v9n2p48
Accepted: April 19, 2020

URL: https://doi.org/10.5430/jha.v9n2p48
Online Published: May 12, 2020

\begin{abstract}
The Extension for Community Health Outcomes (ECHO) project uses teleconferencing to improve access to care for patients who are unable to access academic medical centers. The model can also be used to address key operational issues facing academic medical centers today. Furthermore, the current healthcare landscape is well primed to accept this innovation. We propose application of this model to solve current challenges in healthcare, including: inpatient capacity, network development and access. Applied in these ways, this program has the potential to alleviate stress on hospital systems, ultimately improving patient care and the patient and provider experience.
\end{abstract}

Key Words: Access, Capacity, Telemedicine, Network development

The Extension for Community Health Outcomes (ECHO) project was developed by Dr Sanjeev Aurora to address poor access to specialty services in rural areas. Using providerto-provider videoconferencing, the program links specialists at an academic medical center (AMC) with community providers to engage in case-based discussions. In doing so, ECHO improves access to specialist care for individual patients and creates a learning community: specialists learn about the challenges of care in the community, while primary care doctors improve their knowledge of conditions traditionally treated by specialists. The program has the dual benefit of providing equity in access to care for patients and a supportive community for rural providers who may otherwise experience professional isolation.
The success of the program is evident in is rapid spread to 560 programs in 48 states and 650 programs across 37 countries, ${ }^{[1]}$ spanning a number of diverse medical conditions. The importance of the program was also codified in the signing of the ECHO act by congress in 2016. This act required the Department of Health and Human Services to "report on technology-enabled collaborative learning and capacity building models. . . to facilitate case-based learning, dissemination of best practices, and evaluations of outcomes."[2]

We have applied the ECHO model to improve outcomes for patients discharged from the hospital to post-acute care facilities. ${ }^{[3]}$ Unlike most ECHO programs, our program, called ECHO-Care Transitions (ECHO-CT), centers on a process (the care transition), rather than a specific disease state. Our

*Correspondence: Amber B. Moore; Email: amoore21@mgh.harvard.edu; Address: Massachusetts General Hospital,55 Fruit Street, BLK-1500, Boston, MA 02114, United States. 
hospital-based team connects with affiliated skilled nursing facilities (SNFs) to improve care for recently discharged patients through information-sharing, trouble-shooting and education. This program has improved value by decreasing skilled nursing facility length of stay (LOS), decreasing costs and readmissions, ultimately producing a 9 -fold return on investment. ${ }^{[4]}$

The healthcare landscape has changed since the inception of the program and ECHO can now be used to address key operational issues facing AMCs today, including capacity, network development and access. A number of factors will make uptake of the program easier now than ever before Telemedicine has grown substantially and now has increased acceptance by patients and providers alike as a means for delivering and receiving healthcare. Although the ECHO model has benefits beyond that of traditional phone or video communication in that it creates a bi-directional learning environment and allows for participants to learn from an array of cases presented by others in the conference, improved acceptance of telemedicine is likely to also improve acceptance of ECHO. Medical centers and clinics are now better equipped for videoconferencing, which is becoming a more common form of communication. Furthermore, with newly released billing codes allowing remuneration for services rendered, a better financial argument for expansion now exists. We advocate for exploring new opportunities for ECHOs like ours, which abide by the traditional structure but do not focus on a specific disease state. Additionally, we believe that the traditional ECHO model can be applied in novel ways to address current operational challenges related to inpatient capacity, mergers/network development and access. This paper seeks to describe opportunity for application of the ECHO program in these areas, offering healthcare administrators a new strategy for addressing modern healthcare challenges.

\section{INPATIENT CAPACITY}

Inpatient capacity is a consistent challenge for academic medical centers. Inpatient volume can be improved by decreasing LOS and ensuring that patient needs are well-matched to the expertise and services available at the AMC, ultimately opening hospital beds for patients who need it most. A large portion of excess inpatient days are due to long stay patients; a number of these patients could be served outside of the hospital, but lack viable discharge options. Post-acute care facilities may be reluctant to accept patients due to medical or social complexity. Building relationships and clinical as well as social support through ECHO may help post-acute care staff feel better equipped to take care of these complex patients, thereby facilitating an earlier discharge, reducing LOS and increasing hospital capacity. This type of approach

Published by Sciedu Press could be applied in a number of different disease states, but may be of greatest benefit in conditions associated with social complexity and long LOS, such as intravenous drug use associated infective endocarditis.

As hospital systems expand, there is also an increased focus on triaging patients to the most appropriate care setting. When there is a request to transfer a patient to an AMC, it is often difficult to assemble the expertise needed to make a well-informed decision about the need for transfer. Modeling a transfer process after ECHO-CT has the potential to improve the appropriateness of the transfer, thereby ensuring that patient needs are well-matched to the capabilities of the medical center. This would require a programmatic focus on a process, although individual discussions may focus on a specific disease state. Furthermore, in discussing cases for transfer, community physicians have access to specialty knowledge which may help to improve the appropriateness of future requests, further streamlining the transfer process and ensuring beds are available for those patients who are most in need of tertiary services.

\section{MERGERS AND NETWORK DEVELOPMENT}

In 2017, hospitals nationwide announced a total of 115 mergers, the highest in more than a decade. ${ }^{[5]}$ While hospital mergers are becoming increasingly common in efforts to improve market share, consolidate negotiating power and share financial risk, mergers do not consistently improve value. ${ }^{[6,7]}$ Within a hospital network, disease-specific ECHOs could help to disseminate specialist knowledge from one center to another. Additionally the ECHO program could also be used as we did: to help ease transitions between different areas within a network. In rapidly expanding systems, where there is a risk of depersonalization and standardization is often the goal, ECHO conferences have the potential to create unified teams and processes over a wide geographic spread, while also increasing value.

\section{ACCESS}

In addition to the initial geographic barriers to specialist access noted by Dr Aurora, patients now face increasing wait times to see specialists at AMCs, further limiting access. Efforts to improve access to ambulatory services, including open access, are encouraged by the Agency for Health Care Research and Quality (AHRQ) ${ }^{[8]}$ Wait times at our institutions are often several months, which may negatively impact the quality of care as well as the patient experience. In addition to saving patient's travel time, the ECHO model has the potential to improve access by increasing the number of cases that can be evaluated by a specialist in a given amount of time. When cases are presented to a specialist after ini- 
tial synthesis by a primary provider, a plan for evaluation and treatment can be rendered more quickly than during a direct patient visit. Additionally, by improving primary care provider knowledge, the number or patients that ultimately require consultation may decrease.

Project ECHO has been shown to successfully equip communities with "the right knowledge, at the right place, at the right time." ${ }^{[1]}$ As our hospitals face increasingly complex operational challenges, ECHO has the potentially to allevi- ate stress on hospital systems, improve patient care and the patient and provider experience. Now is the time to promote the reverberation of ECHO across our hospital systems.

\section{ACKNOWLEDGEMENTS}

The authors would like to acknowledge Lauren JungeMaughan for her editorial contributions.

\section{Conflicts of Interest Disclosure}

The authors declare they have no conflicts of interest.

\section{REFERENCES}

[1] Project ECHO. Accessed at February 2, 2020. Available from: https://echo.unm.edu

[2] Congress.gov. Current Legislative activities: S.2873-ECHO Act. Accessed at February 2, 2020. Available from: https ://www . congre ss.gov/bill/114th-congress/senate-bill/2873

[3] Farris G, Sircar M, Bortinger J, et al. Extension for Community Healthcare Outcomes-Care Transitions: enhancing geriatric care transitions through a multidisciplinary videoconference. J Am Geriatr Soc. 2017; 65(3): 598-602. PMid: 28032896. https : //doi.org/ $10.1111 /$ jgs. 14690

[4] Moore A, Krupp JE, Dufour, A, et al. Improving transitions to postacute care for elderly patients using a novel video-conferencing program: ECHO-care transitions. Am J Med. 2017; 130(10): 1199-1204. PMid: 28551043. https ://doi.org/10.1016/j .amjmed. 2017. 04.041
[5] Kaufman Hall. 2017 in Review: The Year M\&A Shook the Healthcare Landscape. Accessed at February 2, 2020. Available from: https://www.kaufmanhall.com/sites/default/file s/legacy_files/2017-in-Review_The-Year-that-Shook -Healthcare.pdf

[6] Beaulieu ND, Dafny LS, Landon BE, et al. Changes in Quality of Care after Hospital Mergers and Acquisitions. N Engl J Med. 2020; 382(1): 51-59. PMid: 31893515. https ://doi .org/10.1056/NE JMsa1901383

[7] Tsai TC, Jha AK. Hospital consolidation, competition and quality: is bigger necessarily better? JAMA. 2014; 213(1): 29-30. PMid: 25058212. https://doi.org/10.1001/jama.2014.4692

[8] AHRQ. The CAHPS Ambulatory Care Improvement Guide. Accessed at February 2, 2020. Available from: https ://www .ahrq.gov/sites/default/files/wysiwyg/c ahps/quality-improvement/improvement-guide/6-strat egies-for-improving/access/cahps-strategy-6a.pdf 